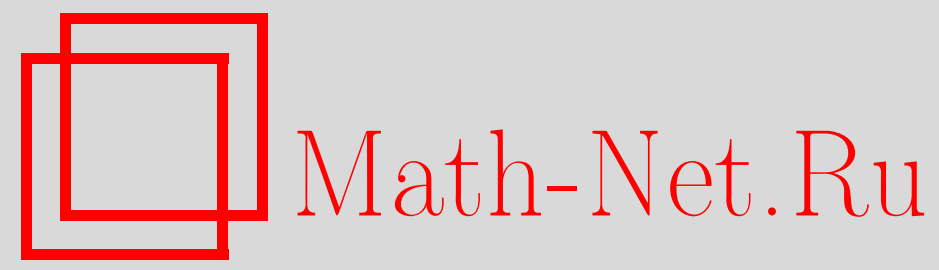

N. James, Y. Peres, Cutpoints and exchangeable events for random walks, Теория вероятн. и ее примен., 1996, том 41, выпуск 4, 854-868

DOI: https://doi.org/10.4213/tvp3239

Использование Общероссийского математического портала Math-Net.Ru подразумевает, что вы прочитали и согласны с пользовательским соглашением http://www.mathnet.ru/rus/agreement

Параметры загрузки:

IP : 3.82 .47 .9

26 апреля 2023 г., 12:52:59

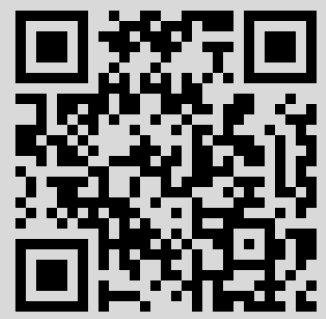




\title{
CUTPOINTS AND EXCHANGEABLE EVENTS FOR RANDOM WALKS ${ }^{1)}$
}

\begin{abstract}
Для марковской цепи $\left\{S_{n}\right\}$ назовем $S_{k}$ точкой разреза (cutpoint) и $k$ - моментом разреза (cut-epoch), если не существует возможных переходов из $S_{i}$ в $S_{j}$ ни при каких $i<k<j$. Покажем, что невозвратное случайное блуждание с ограниченным размером шага на целочисленной решетке имеет бесконечно много точек разреза почти наверное. Для простого случайного блуждания на $\mathbf{Z}^{d}, d \geqslant 4$, этот факт установлен Лолером (Lawler). Далее, пусть $G$ - конечно порожденная группа с по крайней мере полиномиальным порядком роста степени 5, тогда для любого симметричного случайного блуждания на $G$ такого, что шаги имеют ограниченный носитель, который порождает $G$, моменты разреза имеют положительную плотность.

Также будет показано, что для любой марковской цепи, имеющей бесконечно много точек разреза почти наверное, предельные числа размещения (eventual occupation numbers) порождают перестановочную $\sigma$-алгебру. Объединяя эти результаты, можно получить ответ на вопрос, поставленный Каймановичем (Kaimanovich), и частично разрешить предположение Диакониса (Diaconis) и Фридмана (Freedman).
\end{abstract}

Ключевые слова и фразы: точка разреза, перестановочный, цепь Маркова, пуассоновская граница, случайные блуждания на группах.

\section{Introduction}

Let $S_{0}, S_{1}, \ldots$ be a homogenous Markov chain on a countable state space $X$. Considering an event to be a set of trajectories, we say an event $A$ is $e x-$ changeable if it is invariant under finite permutations, i.e., if $\left(s_{0}, s_{1}, \ldots\right) \in A$ then so is $\left(s_{\pi(0)}, \ldots, s_{\pi(n)}, s_{n+1}, \ldots\right)$ for any $n$ and any permutation $\pi$ of $\{0, \ldots, n\}$. A transient process visits each state only finitely often, and so for each state $x$ there is a random variable $V(x)$ which counts the number of visits, $V(x)=\#\left\{n \geqslant 0 \mid S_{n}=x\right\}$. We call the collection $\{V(x)\}_{x \in X}$ the occupation numbers of the process. If an event is defined in terms of these occupation numbers then it is invariant under all permutations,

*Department of Mathematics, 940 Evans Hall, University of California, Berkeley, CA 94720, USA.

** Department of Statistics, 367 Evans Hall, University of California, Berkeley, CA 94720 , USA.

1) Research partially supported by NSF grant \# DMS-9404391. 
of these occupation numbers then it is invariant under all permutations, even infinite ones, and thus is exchangeable. A natural question, posed by Kaimanovich [10], is under what conditions does the converse hold:

For which transient Markov chains is the indicator of every exchangeable event equal a.e. to a function of the occupation numbers?

Kaimanovich [10] showed that this is the case for random walks on $\mathbf{Z}$ with non-zero mean, and explicitly asked about transient mean-zero random walks in higher dimensions.

We now state our main result in greater generality, for random walks on finitely-generated discrete groups. Let $G$ be such a group and let $p$ be a probability measure on $G$ whose support $U$ is finite and generates $G$ as a semigroup. The transition probabilities are then defined by $p(x, y)=$ $p\left(x^{-1} y\right)$ for $x, y$ in $G$. More detailed definitions are given in the next section.

Theorem 1.1. Let $\left\{S_{n}\right\}$ be a transient random walk with bounded increments on the lattice $\mathbf{Z}^{d}$, or a symmetric random walk, with bounded generating support, on a group $G$ that has growth at least polynomial of degree 5 . Then any exchangeable event is given by the occupation numbers, up to a set of measure zero.

The key idea for the proof of this theorem is to apply a sample-path property of transient random walks, the existence of cutpoints. This method also proves a conjecture of Diaconis and Freedman [4], specialized to such random walks; see Corollary 1.2 below. For general transient Markov chains this conjecture, as well as Kaimanovich's question, are still unresolved. A closely related question posed by Kaimanovich and Vershik [11], is still open even for simple random walk on $Z^{3}$ - see Remark 2 at the end of the paper.

Let $S_{0}, S_{1}, \ldots$ be a Markov chain on a countable state space, with transition probabilities $p(x, y)=\mathbf{P}\left\{S_{n+1}=y \mid S_{n}=x\right\}$. The initial distribution of $S_{0}$ is arbitrary. We say there is a permitted transition from $x$ to $y$ if $p(x, y)>0$.

D e f i n i t i o n 1. If there is no permitted transition from $S_{i}$ to $S_{j}$ for all $i$ and $j$ with $0 \leqslant i<n<j$, then $n$ is a cut-epoch for $S$ and $S_{n}$ is a cutpoint.

If $S_{n}$ is a cutpoint then deleting $S_{n}$ from the trajectory cuts the trajectory into two disjoint components, $S[0, n)$ and $S(n, \infty)$, with no possible transition from the first to the second.

In Section 2 we establish the existence of cutpoints. We only consider step distributions $p$ on groups $G$, where the support $U$ of $p$ is finite and generates $G$ as a semigroup.

Theorem 1.2. (a) Any transient random walk with bounded increments on the lattice $\mathbf{Z}^{d}$ has in finitely many cutpoints with probability 1.

(b) If the growth of $G$ is at least polynomial of degree 5, and $p$ is symmetric, then $S_{n}$ is a cutpoint for infinitely many $n$ with probability 1.

A random walk on $\mathbf{Z}^{d}$ can be transient only in two different ways: either the increment distribution $p$ has non-zero mean, or $p$ has mean zero and 
$d \geqslant 3$. In the first case the theorem is easy to prove because it reduces to the case of a one-dimensional walk. For simple random walk on $\mathbf{Z}^{d}$ with $d \geqslant 4$, the existence of cutpoints is essentially proved by Lawler [13] (he considered a related notion of «loop-free» points), but the case $d=3$ is new even for simple random walk. Part (b) and its proof are based on a result of Lawler ([13, Sections 3.2 and 7.7]).

In Section 3 we return to the problem of describing the exchangeable $\sigma$-field. Theorem 1.1 is an immediate consequence of Theorem 1.2 and the following general statement.

Proposition 1.1. Let $\left\{S_{n}\right\}$ be a Markov chain. If $\left\{S_{n}\right\}$ almost surely has infinitely many cutpoints, then the exchangeable $\sigma$-field is generated by the occupation numbers.

In 1955 Hewitt and Savage [7] proved that the exchangeable $\sigma$-field is trivial if $S_{n}$ are i.i.d. Blackwell and Freedman [2] determined the exchangeable $\sigma$-field for recurrent Markov chains; it is atomic, and depends only on the distribution of $S_{0}$. In the case that $\left\{S_{n}\right\}$ is a sequence of independent random variables with a common state space $X$, Aldous and Pitman [1] gave a necessary condition for the exchangeable $\sigma$-field to be trivial, a condition which is sufficient if $X$ is finite. They also gave other sufficient conditions for infinite $X$. Palacios [16] extended their results to some recurrent nonhomogeneous Markov chains.

For transient Markov chains, the exchangeable $\sigma$-field has an alternative representation, described in [9] and [10]. Let $V_{n}(x)=\#\{k \mid 0 \leqslant$ $k \leqslant n$ and $S_{k}=x$ \} denote the number of visits to $x$ up to time $n$. Then $\left\{\left(S_{n}, V_{n-1}\right)\right\}$ is again a Markov chain, whose states are pairs $(x, v)$ where $x \in X$, and $v: X \rightarrow \mathbf{Z}$ vanishes at all but finitely many points. The exchangeable $\sigma$-field of $\left\{S_{n}\right\}$ is exactly the (completed) tail $\sigma$-field of the extended chain $\left\{\left(S_{n}, V_{n-1}\right)\right\}$. We now specialize to the case where $X$ is a group $G$. There is a natural multiplication rule for the states of the extended chain, which makes them into a group. First let $\left(T_{x} v\right)(y)=v\left(x^{-1} y\right)$ for $x, y \in G$ and then the multiplication is $(x, v)(y, w)=\left(x y, v+T_{x} w\right)$. This group is the semi-direct product by $G$ of the direct sum $\bigoplus_{G} \mathbf{Z}$; it is called the wreath product of $\mathbf{Z}$ by $G$, and denoted by $\mathbf{Z}, G$. Let $\delta_{e}: G \rightarrow \mathbf{Z}$ be 1 at the identity $e$ and 0 elsewhere. Given a probability measure $p$ on $G$ we can define a new measure $\mu_{p}$ on $\mathbf{Z} l G$ by $\mu_{p}\left(x, \delta_{e}\right)=p(x)$ for $x \in G$ and $\mu_{p}$ vanishes elsewhere. The process $\left(S_{n}, V_{n-1}\right)$ is now a random walk on $\mathbf{Z}, G$ starting at $\left(S_{0}, 0\right)$, with transition probabilities given by $\mu_{p}$. Applying the general boundary theory in [11] and [10] yields the following consequence of Theorem 1.2 and Proposition 1.1.

Corollary 1.1. If the group $G$ and the step distribution $p$ satisfy the conditions in part (a) or (b) of Theorem 1.2, then the distribution of the occupation field $V$ can be identified with the Poisson boundary for the random walk on $\mathbf{Z}, G$ with step distribution $\mu_{p}$.

While studying extensions of De Finetti's theorem to Markov chains, 
Diaconis and Freedman [4] stated a general conjecture for transient Markov chains $\left\{S_{n}\right\}$. Let $M_{n}(x, y)$ be the number of transitions made from $x$ to $y$ up to time $n$, so that $M_{n}(x, y)$ increases to a finite limit $M(x, y)$ as $n \rightarrow \infty$. They conjectured that the intersection of $\sigma$-fields

$$
\bigcap_{n} \sigma\left(M_{n}, S_{n}, S_{n+1}, S_{n+2}, \ldots\right)
$$

is always generated by $M$.

Corollary 1.2. The intersection $\cap_{n} \sigma\left(M_{n}, S_{n}, S_{n+1}, \ldots\right)$ is generated by $M$ whenever the Markov chain $\left\{S_{n}\right\}$ has infinitely many cutpoints almost surely.

In particular, this applies to the random walks in Theorem 1.2.

$\mathrm{P}$ r o of. The transition counts $M_{n}$ are the visit counts for the 2-block chain $\left\{S_{n}, S_{n+1}\right\}$. Therefore the intersection in the corollary can be identified as the exchangeable $\sigma$-field for the 2-block chain. If $k$ is a cut-epoch for the original chain $\left\{S_{n}\right\}$, then it is clearly also a cut-epoch for the 2-block chain. Hence, since $M$ is the occupation field for the 2-block chain, the assertion of the corollary follows from Proposition 1.1.

\section{Cutpoints}

In this section, $G$ will always be a finitely-generated, infinite discrete group with identity $e$, and $p$ a symmetric probability measure on $G$, whose support $U$ is finite and generates $G$ as a semigroup. The measure $p$ defines transition probabilities by $p(x, y)=p\left(x^{-1} y\right)$ and these are bounded away from zero by $p_{\min }=\min _{u \in U} p(u)$. A point $y$ is a neighbor of $x$ if $y$ is in $x U$. Let $U^{n}=\left\{u_{1} u_{2} \ldots u_{n} \mid u_{i} \in U\right\}$ for $n \geqslant 1$ and $U^{0}=\{e\}$. The union of these sets is $G$. We write $\rho(x)=\min \left\{n \mid x \in U^{n}\right\}$ for $x \in G$.

The ball $U_{n}$ of radius $n$ about the identity in the Cayley graph is $U^{0} U$ $U^{1} \cup \ldots \cup U^{n}$. The group $G$ is of polynomial growth if there exists $d>0$ such that $\# U_{n}=O\left(n^{d}\right)$. In this case the infimum of these $d$ is called the degree of growth of $G$. This does not depend on the choice of generators $U$, but is a property of the group $G$. It is known from work of Gromov [5] that the degree is always an integer.

Let $p_{n}$ be the $n$th convolution power of $p$. The Green function $\theta$, evaluated at a point $x$, is the expected number of visits to $x$ of a random walk starting at $e: \theta(x)=\sum_{n=0}^{\infty} p_{n}(x)$.

Recall that the walk is assumed transient, hence $\theta(x)$ is finite for every $x$. We will need a discrete version of the gradient of the Green function, $\nabla \theta(x)=\max _{y \in x U}|\theta(y)-\theta(x)|$.

We will be interested in the following three hypotheses in the case $d \geqslant 3$ :

$$
\begin{aligned}
\left(A_{0} \rho(x)\right)^{2-d} & \leqslant \theta(x) \leqslant\left(\frac{\rho(x)}{A_{1}}\right)^{2-d} \\
& \text { for all } x \in G \text { for some } A_{0}, A_{1} ;
\end{aligned}
$$




$$
\begin{aligned}
\nabla \theta(x) & \leqslant\left(\frac{\rho(x)}{A_{2}}\right)^{1-d} \quad \text { for all } \quad x \in G \text { for some } A_{2} \\
\left(A_{3} \rho(x)\right)^{1-d} & \leqslant \nabla \theta(x) \\
& \text { for all but finitely many } x \in G \text { for some } A_{3} .
\end{aligned}
$$

In the proposition below we shall exhibit a particular kind of cutpoint. If the random walk $\left\{S_{n}\right\}$ starts inside some sphere and visits that sphere only once, at time $n$ say, then $n$ is a cut-epoch for $S$, because deleting $S_{n}$ disconnects the past of the trajectory, which is inside the sphere, from the future, which is outside. This approach works nicely to prove existence of approximate cut-spheres for Brownian motion (see Proposition 1 in [3]) but in the generality of Theorem 1.2 more work is required to obtain the necessary estimates. The obvious choice for such a sphere is the set of points at some fixed distance from the identity, but we find it easier to work with sets on which the Green function is almost constant. For the rest of this section, given $d, A_{0}$ and $A_{2}$ we take

$$
0<\eta<d-2 \text { and } \delta=\eta\left(A_{0} A_{2}\right)^{1-d}
$$

For $k \geqslant 1$ we define

$$
\begin{aligned}
B_{k} & =\left\{x \in G \mid \theta(x) \geqslant\left(\frac{\delta}{k}\right)^{d-2}\right\} \text { and } \\
\partial B_{k} & =\left\{x \in B_{k} \mid x U \text { contains some } y \notin B_{k}\right\} .
\end{aligned}
$$

The difference set $B_{k} \backslash \partial B_{k}$ is called the interior of $B_{k}$. If $x$ is in the interior of $B_{k}$ and $y \notin B_{k}$, then clearly every path from $x$ to $y$ intersects $\partial B_{k}$.

Let cut $_{k}$ be the event that $\left\{S_{n}\right\}$ starts in the interior of $B_{k}$ and visits $\partial B_{k}$ exactly once:

$$
\begin{gathered}
\text { cut }_{k}=\bigcup_{n=1}^{\infty}\left\{S_{i} \in B_{k} \backslash \partial B_{k} \text { for } i<n, S_{n} \in \partial B_{k},\right. \text { and } \\
\left.S_{j} \notin B_{k} \text { for } j>n\right\} .
\end{gathered}
$$

Proposition 2.1. If $G$ is of polynomial growth of degree $d \geqslant 3$ and (1)-(3) hold then with probability 1 , the events cut $_{k}$ happen for infinitely many $k$.

In Theorem 5.1 of [6], Hebisch and Saloff-Coste show that if $p$ is symmetric then (1), (2) hold without any further conditions on $G$.

Corollary 2.1. If the measure $p$ is symmetric, the group $G$ is of polynomial growth of degree $d \geqslant 3$, and (3) holds, then with probability 1 , the events cut $_{k}$ happen for infinitely many $k$.

The proof of Proposition 2.1 is based on the second moment method and the following three lemmas. The first lemma separates the spheres $\partial B_{k}$ for distinct $k$, the second compares hitting probabilities of two spheres for 
the walk started in the shell between them, and the third lemma bounds the maximal distance from a point $x \in \partial B_{k}$ to $\partial B_{k+1}$.

Lemma 2.1. Let $r_{k}=(\delta / k)^{d-2}$ and $R_{k}=(1+\eta / k)(\delta / k)^{d-2}$. If (1), (2) hold then for all $k \geqslant 1$ and $x \in \partial B_{k}$, we have $r_{k} \leqslant \theta(x) \leqslant R_{k}$.

$\mathrm{P}$ r o o f. Let $x \in \partial B_{k}$ and let $y \notin B_{k}$ be a neighbor of $x$, i.e. $y \in x U$. Then (1) and (4) yield $\left(A_{0} \rho(y)\right)^{2-d} \leqslant \theta(y)<(k / \delta)^{2-d}$ hence $\rho(y)>k /\left(\delta A_{0}\right)$, and by (2) we get $\nabla \theta(y) \leqslant\left(\delta A_{0} A_{2} / k\right)^{d-1}$. An upper bound for $\theta(x)$ is

$$
\theta(x) \leqslant \theta(y)+\nabla \theta(y) \leqslant\left(1+\frac{\delta\left(A_{0} A_{2}\right)^{(d-1)}}{k}\right)\left(\frac{\delta}{k}\right)^{d-2}=R_{k} .
$$

We note the following facts about these bounds:

$$
\begin{aligned}
& r_{k}-R_{k+1} \sim \delta^{d-2} \frac{d-2-\eta}{k^{d-1}} \quad \text { as } \quad k \rightarrow \infty \text {; } \\
& R_{k}-r_{k+1} \sim \delta^{d-2} \frac{d-2+\eta}{k^{d-1}} \quad \text { as } \quad k \rightarrow \infty \text {; } \\
& 0<r_{k}-r_{m} \leqslant \delta^{d-2}(d-2) \frac{m-k}{k^{d-2} m} \quad \text { for } \quad 1 \leqslant k<m \text {; } \\
& R_{k}-R_{m} \geqslant \delta^{d-2} \frac{m-k}{k^{d-2} m}>0 \quad \text { for } \quad 1 \leqslant k<m .
\end{aligned}
$$

Here the symbol $\sim$ means that the ratio of the two sides tends to 1 . The last two inequalities follow from the expansion

$$
\left(\frac{1}{k}\right)^{d-2}-\left(\frac{1}{m}\right)^{d-2}=\left(\frac{1}{k}-\frac{1}{m}\right) \sum_{j=0}^{d-3}\left(\frac{1}{k}\right)^{j}\left(\frac{1}{m}\right)^{d-3-j}
$$

The asymptotic relation (6) implies that for $k$ sufficiently large, $r_{k}-$ $R_{k+1}>0$, and hence the spheres $\partial B_{k}$ and $\partial B_{k+1}$ are disjoint.

Lemma 2.2. If (1), (2) hold then there exist a real $C$ and an integer $K \geqslant 1$ such that for all $K \leqslant k<m$ and all starting points $x \in \partial B_{k+1}$,

$$
\frac{m}{C k(m-k)} \leqslant \mathrm{P}_{x}\left\{\left\{S_{n}\right\} \text { visits } \partial B_{m} \text { before } \partial B_{k}\right\} \leqslant \frac{C m}{k(m-k)}
$$

and consequently for all $x \in \partial B_{k+1}$,

$$
\frac{1}{C k} \leqslant \mathbf{P}_{x}\left\{\left\{S_{n}\right\} \text { never visits } \partial B_{k}\right\} \leqslant \frac{C}{k} .
$$

P r o of. Let $h(x)=\mathbf{P}_{x}\left\{\left\{S_{n}\right\}\right.$ visits $\partial B_{m}$ before $\left.\partial B_{k}\right\}$. The function $h$ is $p$-harmonic in the shell $B_{m} \backslash\left(\partial B_{m} \cup B_{k}\right)$. It has boundary values 0 on $\partial B_{k}$ and 1 on $\partial B_{m}$. The Green function $\theta$ is $p$-harmonic at all points except the identity. Let

$$
u(x)=\frac{r_{k}-\theta(x)}{r_{k}-r_{m}} \quad \text { and } \quad U(x)=\frac{R_{k}-\theta(x)}{R_{k}-R_{m}} .
$$


It follows from Lemma 2.1 that $u(x) \leqslant h(x) \leqslant U(x)$ for $x \in \partial B_{k} \cup \partial B_{m}$. The maximum principle for $p$-harmonic functions then forces these inequalities to extend to $x \in B_{m} \backslash B_{k}$. For $x \in \partial B_{k+1}$ we infer that

$$
u(x) \geqslant \frac{r_{k}-R_{k+1}}{r_{k}-r_{m}} \quad \text { and } \quad U(x) \leqslant \frac{R_{k}-r_{k+1}}{R_{k}-R_{m}} .
$$

The assertion (10) is now implied by (6)-(9), and the inequalities (11) follow by letting $m \rightarrow \infty$.

The following lemma says that for each point $x \in \partial B_{k}$ there is a path starting at $x$ and leading to $\partial B_{k+1}$ which does not re-enter $\partial B_{k}$, and the length of the path can be bounded uniformly in $x$ and $k$.

Lemma 2.3. If (1)-(3) hold then there are integers $J$ and $k_{0}$ such that for any $k \geqslant k_{0}$ and any $x \in \partial B_{k}$ there is a finite path $x_{0}=x, x_{1}, \ldots, x_{j}$ in $G$ of length $j \leqslant J$ that terminates in $x_{j} \in \partial B_{k+1}$, such that $x_{i} \in x_{i-1} U$ and $x_{i} \notin \partial B_{k}$ for $i=1, \ldots, j$.

$\mathrm{P}$ r o of. Choose $k_{0}$ so large that the inequality in (3) holds for $x$ outside $B_{k_{0}}$. Let $k \geqslant k_{0}$ and $x \in \partial B_{k}$. The definition of $\partial B_{k}$ implies that $x$ has a neighbor $y \in x U$ which is outside $B_{k}$. Let $x_{1}=y$. We now follow the line of steepest descent of the Green function. Having defined $x_{i}$ choose $x_{i+1} \in x_{i} U$ such that $\theta\left(x_{i+1}\right)=\min _{z \in x_{i} U} \theta(z)$. Since $\theta$ is $p$ harmonic and $\theta\left(x_{0}\right)>\theta\left(x_{1}\right)$, the sequence $\left\{\theta\left(x_{i}\right)\right\}_{i \geqslant 0}$ is strictly decreasing. The group $G$ is discrete and $\theta$ vanishes at $\infty$ so $\theta\left(x_{i}\right) \downarrow 0$. Choose $j$ such that $\theta\left(x_{j}\right) \geqslant r_{k+1}>\theta\left(x_{j+1}\right)$.

We now give an upper bound for $j$. Obviously

$$
(j-1) \min _{i<j}\left(\theta\left(x_{i}\right)-\theta\left(x_{i+1}\right)\right) \leqslant \theta\left(x_{1}\right)-\theta\left(x_{j}\right) .
$$

Each $x_{i}$ has a neighbor $w \in x_{i} U$ such that $\theta(w)$ differs from $\theta\left(x_{i}\right)$ by $\nabla \theta\left(x_{i}\right)$. If $\theta(w)$ is greater than $\theta\left(x_{i}\right)$ then the (weighted) average of $\theta$ over the other neighbors of $x_{i}$ must be correspondingly less, and it follows from the definition of $x_{i+1}$ that $\theta\left(x_{i}\right)-\theta\left(x_{i+1}\right) \geqslant p_{\min } \nabla \theta\left(x_{i}\right)$. Since $x_{i} \in B_{k+1}$ for $i \leqslant j$, we have

$$
\left(\frac{\delta}{k+1}\right)^{d-2} \leqslant \theta\left(x_{i}\right) \leqslant\left(\frac{A_{1}}{\rho\left(x_{i}\right)}\right)^{d-2}
$$

where the right-hand inequality is from (1). Therefore $\rho\left(x_{i}\right) \leqslant A_{1}(k+1) / \delta$, and $\nabla \theta\left(x_{i}\right) \geqslant\left(A_{1} A_{3}(k+1) / \delta\right)^{1-d}$ by (3). On the other hand

$$
\theta\left(x_{1}\right)-\theta\left(x_{j}\right) \leqslant r_{k}-r_{k+1} \leqslant \delta^{d-2}(d-2) k^{1-d}
$$

by (8). Substituting this into (12) gives

$$
(j-1) p_{\min }\left(\frac{A_{1} A_{3}(k+1)}{\delta}\right)^{1-d} \leqslant \delta^{d-2}(d-2) k^{1-d} .
$$

Recalling that $\delta=\eta\left(A_{0} A_{2}\right)^{1-d}$, we conclude that

$$
j \leqslant J=1+2^{d-1}\left(A_{0} A_{1} A_{2} A_{3}\right)^{d-1} \frac{d-2}{\eta p_{\min }} \quad \text { for all } k \geqslant k_{0} .
$$


$\mathrm{Pr}$ o of of $\mathrm{Proposition} 2.1$. As in the preceding Lemma, choose $k_{0}$ so that the inequality (3) holds for $x$ outside $B_{k_{0}}$. Let $k \geqslant k_{0}$. One way in which a trajectory (starting from the interior of $B_{k}$ ) can be in cut ${ }_{k}$ is that it moves randomly until it reaches $\partial B_{k}$, then marches out to $\partial B_{k+1}$, and never visits $\partial B_{k}$ again. Now the probability of reaching $\partial B_{k}$ is 1 . According to Lemma 2.3, from each $x \in \partial B_{k}$ there is a path of length at most $J$, which leads to $\partial B_{k+1}$ without re-entering $\partial B_{k}$. Thus the probability of going from $x \in \partial B_{k}$ to $\partial B_{k+1}$ without revisiting $\partial B_{k}$ is at least $p_{\min }^{J}$. Therefore, using the lower bound in (11), we obtain $\mathbf{P}\left(\operatorname{cut}_{k}\right) \geqslant p_{\min }^{J} /(C k)$. On the other hand, every trajectory in cut $_{k}$ must visit $\partial B_{k+1}$ and never return to $\partial B_{k}$, hence by the upper bound in (11), $\mathbf{P}\left(\right.$ cut $\left._{k}\right) \leqslant C / k$.

Let $k_{0} \leqslant k<m$ and consider a trajectory in the event cut $_{k} \cap \mathrm{cut}_{m}$. It visits $\partial B_{k}$, then some point $x$ in $\partial B_{k+1}$ and from $x$ it reaches $\partial B_{m}$ without revisiting $\partial B_{k}$. After reaching $\partial B_{m+1}$ it never re-enters $B_{m}$. Thus by Lemma 2.2 ,

$$
\begin{aligned}
\mathbf{P}\left\{\text { cut }_{k} \cap \text { cut }_{m}\right\} \leqslant & \max _{x \in \partial B_{k+1}} \mathbf{P}_{x}\left\{\left\{S_{n}\right\} \text { visits } \partial B_{m} \text { before } \partial B_{k}\right\} \\
& \times \max _{y \in \partial B_{m+1}} \mathbf{P}_{y}\left\{(\forall n) S_{n} \notin B_{m}\right\} \\
\leqslant & \frac{C m}{k(m-k)} \frac{C}{m}=\frac{C^{2}}{k(m-k)} .
\end{aligned}
$$

It follows that

$$
\sum_{k=k_{0}}^{K} \mathbf{P}\left(\mathrm{cut}_{k}\right) \geqslant \sum_{k=k_{0}}^{K} \frac{p_{\min }^{J}}{C k} \sim \frac{p_{\min }^{J}}{C} \log K
$$

and

$$
\sum_{k, m=k_{0}}^{K} \mathbf{P}\left(\mathrm{cut}_{k} \cap \mathrm{cut}_{m}\right) \leqslant 2 \sum_{k_{0} \leqslant k<m \leqslant K} \frac{C^{2}}{k(m-k)}+\sum_{k=k_{0}}^{K} \frac{C}{k} \sim 2 C^{2} \log ^{2} K .
$$

By the Kochen-Stone Lemma [12],

$$
\mathbf{P}\left(\text { cut }_{k} \text { i.o. }\right) \geqslant \limsup _{K \rightarrow \infty} \frac{\left(\sum_{k=1}^{K} \mathbf{P}\left(\operatorname{cut}_{k}\right)\right)^{2}}{\sum_{k, m=1}^{K} \mathbf{P}\left(\operatorname{cut}_{k} \cap \operatorname{cut}_{m}\right)} \geqslant \frac{p_{\min }^{2 J}}{2 C^{4}} .
$$

Now suppose the first $n$ steps of the walk are specified. We may choose $k_{0}$ large enough so that $e, x_{1}, \ldots, x_{n}$ all lie in the interior of $B_{k_{0}}$ and repeat the reasoning above to obtain

$$
\mathbf{P}\left\{\operatorname{cut}_{k} \text { i.o. } \mid S_{0}=e, \ldots, S_{n}=x_{n}\right\} \geqslant \frac{p_{\min }^{2 J}}{2 C^{4}} .
$$

In other words the martingale $\mathbf{E}\left(\mathbf{1}_{\left\{\text {cut }_{k} \text { i.o. }\right\}} \mid S_{0}, \ldots, S_{n}\right)$, which converges a.s. to $\mathbf{1}_{\left\{\text {cut }_{k} \text { i.o. }\right\}}$, is bounded away from zero. Therefore $\mathbf{P}\left(\mathrm{cut}_{k}\right.$ i.o. $)=1$. 
P r o of of $\mathrm{T}$ h e or e m 1.2. (a) First we consider the case that $p$ has non-zero mean. Suppose there is a homomorphism of a group $G$ onto another group $G^{\prime}$ which maps $p$ to $p^{\prime}$, and $S_{n}$ to $S_{n}^{\prime}$. If $S_{n}^{\prime}$ is a cutpoint then so is $S_{n}$. The mean of $p$ has a component which is non-zero and we may take our homomorphism to be the projection onto that component. This gives a random walk $S_{n}^{\prime}$ on $\mathbf{Z}$ of bounded stepsize with non-zero drift. Using the ergodic theorem, it is easy to see that this walk has infinitely many cutpoints a.s. See part (b), where it is shown that a decay assumption (14) on the convolution powers of $p$ implies the existence of cutpoints; this assumption is certainly satisfied for walks with non-zero mean.

Now suppose $p$ has mean zero. Then $d$ must be at least 3 for the walk to be transient. By Proposition 2.1, it suffices to show that the Green function satisfies (1)-(3). This will follow from a bound on the error in the local central limit theorem for random walks on $\mathbf{Z}^{d}$ due to Lawler and Polaski [14]. Let $\Sigma$ be the covariance matrix of $p$, i.e., $\Sigma=\left(\mathbf{E}\left[Y^{(i)} Y^{(j)}\right]\right)$ where $Y=\left(Y^{(1)}, \ldots, Y^{(d)}\right)$ has distribution $p$. For $x \in \mathbf{Z}^{d}$ we write $\|x\|=$ $\left(x \cdot \Sigma^{-1} x\right)^{1 / 2}$. Let $b=(1 / 2) \Gamma(d / 2-1) \pi^{-d / 2}(\operatorname{det} \Sigma)^{-1 / 2}$. In our case $p$ has finite moments of all orders, but the lemma below holds whenever $p$ has a finite $(4 d-6)$ th moment, and this moment assumption could be weakened with more work.

Lemma 2.4. We have

$$
\theta(x)=b\|x\|^{2-d}+o\left(\|x\|^{1-d}\right) .
$$

Section 3 (p. 796-799) of [14] states this with an error term $O\left(\|x\|^{1-d}\right)$ under the weaker assumption that $p$ has a finite $(4 d-8)$ th moment, but their work contains a range of bounds on the error depending on how many moments of $p$ are assumed to be finite. We explain briefly how the above lemma follows from their estimates.

We may assume $p$ is strongly aperiodic. (See $[17$, p. 310] for details of this reduction.) Recall that $p_{n}$ denotes the $n$th convolution power of $p$. We approximate $p_{n}(x)$ by

$$
\widetilde{p}_{n}(x)=(2 \pi n)^{-d / 2}(\operatorname{det} \Sigma)^{-1 / 2} \exp \left\{-\frac{\|x\|^{2}}{2 n}\right\}
$$

for $n \geqslant 1$, and let $\widetilde{p}_{0}$ be the point mass at the origin. Now

$$
\left|\theta(x)-b\|x\|^{2-d}\right| \leqslant \sum_{n=0}^{\infty}\left|p_{n}(x)-\widetilde{p}_{n}(x)\right|+\left|\sum_{n=0}^{\infty} \widetilde{p}_{n}(x)-b\|x\|^{2-d}\right| .
$$

The last term is $O\left(\|x\|^{-d}\right)$. The first term can be split into three parts:

$$
\left(\sum_{0 \leqslant n<\|x\|^{a}}+\sum_{\|x\|^{a} \leqslant n<\|x\|^{2}}+\sum_{\|x\|^{2} \leqslant n}\right)\left|p_{n}(x)-\widetilde{p}_{n}(x)\right|,
$$


where $a<2$ is an arbitrary constant, to be chosen below. Lawler and Polaski show the last sum in (13) is $O\left(\|x\|^{-d}\right)$ and the second sum is $O\left(\|x\|^{-\gamma}\right)$, where $\gamma=[4+a(d-2)] / 2$. We will choose $a$ such that $2(d-3) /(d-2)<a<2$, hence $\gamma>d-1$. In the first sum in (13), $\sum_{0 \leqslant n<\|x\|^{a}} \widetilde{p}_{n}(x)$ is negligible because $a<2$. Lemma 3.2 of [14], which follows from Theorems 2.1 and 2.3 of Ney and Spitzer [15], show that if $p$ has finite $(2 m)$ th moment ( $m$ an integer) then

$$
\sum_{0 \leqslant n<\|x\|^{a}} p_{n}(x)=O\left(\|x\|^{-\beta}\right), \quad \text { where } \quad \beta=m\left(1-\frac{a}{2}\right)+\frac{(d-2) a}{2} .
$$

Choosing $a$ close to $2(d-3) /(d-2)$ yields $\beta>d-1$, since $m>2(d-2)$. Thus the error term is $o\left(\|x\|^{1-d}\right)$, and this completes the proof the lemma.

$\mathrm{Pr}$ o of of $\mathrm{Th}$ e o r e m 1.2, con t in u ed. If $u \in \mathbf{Z}^{d}$ is fixed, then

$$
\|x+u\|^{2-d}=\|x\|^{2-d}+O\left(\|x\|^{1-d}\right),
$$

hence

$$
\nabla \theta(x)=\max _{u \in U}|\theta(x+u)-\theta(x)|=O\left(\|x\|^{1-d}\right) .
$$

Because $U$, the support of $p$, generates $\mathbf{Z}^{d}$ as a semigroup, there is a constant $c>0$ such that for all $x \in \mathbf{Z}^{d}$ we can choose $u_{x} \in U$ such that $u_{x} \cdot \Sigma^{-1} x \geqslant$ $c\|x\|$. Now

$$
\begin{aligned}
\left\|x+u_{x}\right\|^{2-d} & =\left(\|x\|^{2}+2 u_{x} \cdot \Sigma^{-1} x+\left\|u_{x}\right\|^{2}\right)^{1-d / 2} \\
& \leqslant\|x\|^{2-d}\left(1+2 c\|x\|^{-1}\right)^{1-d / 2} \\
& =\|x\|^{2-d}\left(1-(d-2) c\|x\|^{-1}+o\left(\|x\|^{-1}\right)\right) .
\end{aligned}
$$

Thus

$$
\theta(x)-\theta\left(x+u_{x}\right) \geqslant b(d-2) c\|x\|^{1-d}+o\left(\|x\|^{1-d}\right) .
$$

Now $\rho(\cdot)$ and $\|\cdot\|$ are equivalent, each being bounded by a multiple of the other, hence (1)-(3) hold.

(b) For symmetric $p$ with bounded generating support, Varopoulos [18], [19] has shown that the growth of $G$ is at least polynomial of degree 5 if and only if the convolution powers $p_{n}$ satisfy

$$
p_{n}(e)=O\left(n^{-5 / 2}\right) \text {. }
$$

We will need only (14) to show the existence of cutpoints, and (14) holds for many asymmetric walks as well. Of course $p_{n}(x)$ decays at the same rate for any fixed $x$.

We may assume that $S_{0}=e$. We extend $S_{n}$ to be doubly infinite. Let $X_{n}=S_{n-1}^{-1} S_{n}, n \geqslant 1$, be the increments of the walk. These are i.i.d. with common distribution $p$. Choose $X_{n}, n \leqslant 0$, to be i.i.d. with distribution $p$ and independent of $X_{n}, n \geqslant 1$. For $n \geqslant 1$ we define $S_{-n}=X_{0}^{-1} \ldots X_{-n+1}^{-1}$. 
Recall $n$ is a cutpoint for $S$ if $S[0, n) U \cap S(n, \infty)=\varnothing$. Lawler [13] discusses loop-free points; the time $n$ is loop-free for $S$ if $S(-\infty, n] \cap S(n, \infty)=$ $\varnothing$. These are obviously similar to cutpoints, and the following argument is based on the method used to establish the existence of loop-free points in Sections 3.2 and 7.7 of [13].

We will show that

$$
\mathbf{P}\{S(-\infty, n) U \cap S(n, \infty)=\varnothing \text { for infinitely many } n \geqslant 1\}=1 .
$$

First we show that the event

$$
D_{n}=\{S(-\infty, n) U \cap S(n, \infty)=\varnothing\}
$$

has positive probability. This probability is independent of $n$ and so we take $n=0$.

Let

$$
\mathcal{N}=\sum_{i, j=0}^{\infty} \mathbf{1}_{\left\{S_{j} \in S_{-i} U\right\}}
$$

count the number of pairs $(i, j)$ such that $i, j \geqslant 0$ and there is a possible transition from $S_{-i}$ to $S_{j}$. Clearly $\mathcal{N} \geqslant 1$ (consider $(i, j)=(1,0)$ ). The condition on $p$ in (14) entails

$$
\mathbf{E} \mathcal{N}=\sum_{i, j=0}^{\infty} \mathbf{P}\left\{S_{-i}^{-1} S_{j} \in U\right\}=\sum_{i, j=0}^{\infty} \sum_{u \in U} p_{i+j}(u) \leqslant C \sum_{i, j=0}^{\infty}(i+j+1)^{-5 / 2}<\infty
$$

for some finite $C$. Hence $\mathcal{N}$ is finite a.s.

We define a pair $\left(i_{0}, j_{0}\right)$ of non-negative integers to be $*$-last if $S_{j_{0}}$ is in $S_{-i_{0}} U$ and $S_{j}$ is not in $S_{-i} U$ for $i \geqslant i_{0}+1, j \geqslant j_{0}$. There is at least one such pair: choose $i_{0}$ maximal so that there is a $j$ with $S_{j} \in S_{-i_{0}} U$ and then choose $j_{0}$ maximal so that $S_{j_{0}} \in S_{-i_{0}} U$. Let $\mathcal{N}_{*}$ count the number of $*$-last pairs. We have

$$
1 \leqslant \mathbf{E} \mathcal{N}_{*}=\sum_{i_{0}, j_{0}=0}^{\infty} \mathbf{P}\left\{S_{-i_{0}}^{-1} S_{j_{0}} \in U \text { and } S_{-i}^{-1} S_{j} \notin U \text { for } i \geqslant i_{0}+1, j \geqslant j_{0}\right\} .
$$

We condition on the value of $S_{-i_{0}}^{-1} S_{j_{0}}$ and use stationarity to obtain

$$
\begin{aligned}
\mathbf{E} \mathcal{N}_{*} & =\sum_{i_{0}, j_{0}=0}^{\infty} \sum_{u \in U} p_{i_{0}+j_{0}}(u) \mathbf{P}\left\{S_{-i}^{-1} u S_{j} \notin U \text { for } i \geqslant 1, j \geqslant 0\right\} \\
& \leqslant \mathbf{E} \mathcal{N} \max _{u \in U} \mathbf{P}\left\{S_{-i}^{-1} u S_{j} \notin U \text { for } i \geqslant 1, j \geqslant 0\right\} .
\end{aligned}
$$

At this point we modify the argument in Section 7.7 of [13] to deal with the maximum over $U$. Let $Y$ have distribution $p$ and be independent 
of $X_{n},-\infty<n<\infty$. Note that $\left\{S_{-i}^{-1} S_{j}: i \geqslant 1, j \geqslant 1\right\}$ has the same distribution as $\left\{S_{-i}^{-1} Y S_{j-1}, i \geqslant 1, j \geqslant 1\right\}$. Replacing $j-1$ by $j$ gives

$$
\mathbf{P}\left(D_{0}\right)=\mathbf{P}\left\{S_{-i}^{-1} Y S_{j} \notin U \text { for } i \geqslant 1, j \geqslant 0\right\} \text {. }
$$

By conditioning on the value of $Y$ we obtain

$$
\mathbf{P}\left(D_{0}\right) \geqslant p_{\min } \max _{u \in U} \mathbf{P}\left\{S_{-i}^{-1} u S_{j} \notin U \text { for } i \geqslant 1, j \geqslant 0\right\} \text {. }
$$

Hence

$$
\mathbf{P}\left(D_{0}\right) \geqslant p_{\min } \frac{\mathbf{E} \mathcal{N}_{*}}{\mathbf{E} \mathcal{N}} \geqslant \frac{p_{\min }}{\mathbf{E} \mathcal{N}}>0
$$

We can now apply the ergodic theorem because $\left(X_{n}\right)_{n=-\infty}^{\infty}$ is an ergodic, stationary sequence. With probability 1

$$
\frac{1}{N} \sum_{n=1}^{N} \mathbf{1}_{D_{n}} \rightarrow \mathbf{P}\left(D_{0}\right)
$$

Thus the set $\{n \geqslant 1 \mid n$ is a cut-epoch for $S\}$ almost surely has positive density which is at least $p_{\min } / \mathbf{E N}$.

\section{Exchangeable events}

We return to the setting where $S_{0}, S_{1}, \ldots$ is a transient Markov chain on the state space $X$. Recall that

$$
V_{n}(x)=\sum_{i=0}^{n} 1_{S_{i}}(x)
$$

and

$$
V(x)=\lim _{n \rightarrow \infty} V_{n}(x)
$$

for every $x \in X$.

Lemma 3.1. For $n \geqslant 1$ consider the event $\mathcal{C}_{n}=\left\{S_{n}\right.$ is a cutpoint $\}$. For $n \geqslant 1$ this event is in the $\sigma$-field $\sigma\left(V, S_{0}\right)$, and for trajectories in $\mathcal{C}_{n}$ the values of $V_{n-1}$ and $S_{n}$ are determined by the values of $V$ and $S_{0}$.

P r o of. Let $\left(s_{0}, s_{1}, \ldots\right)$ be a trajectory with only permitted transitions and let $v=\mathbf{1}_{s_{0}}+\mathbf{1}_{s_{1}}+\cdots$ be the value of $V$ for this trajectory. If $\left(s_{0}, s_{1}, \ldots\right)$ lies in $\mathcal{C}_{n}$, then $v$ may be decomposed as

$$
v=v_{1}+\mathbf{1}_{y}+v_{2}
$$

where $v_{1}=\mathbf{1}_{s_{0}}+\cdots+\mathbf{1}_{s_{n-1}}$, in the middle term $y=s_{n}$, and $v_{2}=\mathbf{1}_{s_{n+1}}+$ $\mathbf{1}_{s_{n+2}}+\cdots$. This decomposition has the following properties:

1. $v_{1}(y)=v_{2}(y)=0, \quad v_{1}\left(s_{0}\right)>0$

2. $\sum_{x \in X} v_{1}(x)=n$;

3. if $v_{1}\left(z_{1}\right)>0$ and $v_{2}\left(z_{2}\right)>0$, then $p\left(z_{1}, z_{2}\right)=0$. 
To establish the first property, we note if $s_{i}=s_{n}$ for some $i<n$ then $p\left(s_{i}, s_{n+1}\right)=p\left(s_{n}, s_{n+1}\right)>0$, and we reason similarly if $v_{2}\left(s_{n}\right)>0$. Properties 2 and 3 are obvious.

Conversely, suppose a decomposition $v=v_{1}+1_{y}+v_{2}$ with properties 1-3 is given. We show $v_{1}=\mathbf{1}_{s_{0}}+\cdots+\mathbf{1}_{s_{n-1}}, y=s_{n}$ and $v_{2}=\mathbf{1}_{s_{n+1}}+\mathbf{1}_{s_{n+2}}+\cdots$, and then Property 3 implies $\left(s_{0}, s_{1}, \ldots\right)$ lies in $\mathcal{C}_{n}$. Now $y=s_{k}$ for some $k$, which is unique by Property 1 . Choose $i_{1}, \ldots, i_{n-1}$ such that

$$
v_{1}=1_{s_{0}}+1_{s_{i_{1}}}+\cdots+1_{s_{i_{n-1}}} .
$$

The only arrangement consistent with Property 3 is that $\left\{i_{1}, \ldots, i_{n-1}\right\}=$ $\{1, \ldots, n-1\}$ and $k=n$.

Thus $\mathcal{C}_{n}$ may be partitioned into subevents $\mathcal{C}_{n} \cap\left\{V_{n-1}=v_{1}, S_{n}=y\right\}$ which are in the $\sigma$-field $\sigma\left(V, S_{0}\right)$, and the lemma is proved.

$\mathrm{Pr}$ o of of $\mathrm{Prop}$ os it i on 1.1. First suppose that the distribution of $S_{0}$ is concentrated at one state, i.e., $\mathbf{P}\left\{S_{0}=x_{0}\right\}=1$ for some $x_{0} \in X$. Then we can describe the conditional distribution of the process given the occupation field $V$. By hypothesis there is an infinite sequence of cut-epochs $n_{1}<n_{2}<\cdots$; by Lemma 3.1 , at these cut-epochs the values of $S_{n_{1}}, S_{n_{2}}, \ldots$ are determined. The sequence of states $\left(S_{n_{k}}, \ldots, S_{n_{k+1}-1}\right)$ visited between succesive cut-epochs is constrained to start at $S_{n_{k}}$ and have occupation numbers $V_{n_{k+1}-1}-V_{n_{k}-1}$. For different $k$ these sequences are mutually independent, and hence the tail $\sigma$-field of the process $\left\{S_{n}, V_{n-1}\right\}_{n \geqslant 0}$, given $V$, is trivial by the Kolmogorov $0-1$ law. It then follows (see Theorem 1.2 in [10]) that the tail $\sigma$-field of the extended chain, which is identical to the exchangeable $\sigma$-field of the original chain, is generated by the occupation field $V$.

Now suppose $S_{0}$ is random. Consider two trajectories, $\left(x_{0}, x_{1}, \ldots\right)$ and $\left(y_{0}, y_{1}, \ldots\right)$, with the same occupation numbers. Then $y_{0}=x_{m}$ for some $m$. If $n>m$ is a cut-epoch for $\left(x_{0}, x_{1}, \ldots\right)$, then $n$ is also a cut-epoch for $\left(y_{0}, y_{1}, \ldots\right)$, we have $x_{n}=y_{n}$, and the initial segments $\left(x_{0}, \ldots, x_{n-1}\right)$ and $\left(y_{0}, \ldots, y_{n-1}\right)$ have the same occupation numbers. Indeed let

$$
\begin{aligned}
& i=\max \left\{k \mid y_{k} \in\left\{x_{0}, \ldots, x_{n-1}\right\}\right\}, \text { and } \\
& j=\min \left\{k \mid y_{k} \in\left\{x_{n+1}, x_{n+2}, \ldots\right\}\right\} .
\end{aligned}
$$

Then $y_{j} \neq x_{m}=y_{0}$ so $j>0$. If $y_{j-1} \in\left\{x_{0}, \ldots, x_{n-1}\right\}$ then $x_{n}$ is not a cutpoint. Thus $y_{j-1}=x_{n}$, and similarly $y_{i+1}=x_{n}$. Hence $y_{n}=x_{n}$ and $\left(y_{0}, \ldots, y_{n-1}\right)$ is a rearrangement of $\left(x_{0}, \ldots, x_{n-1}\right)$. This shows that for the conditioned extended chain there is a sequence of times $n_{1}<n_{2}<\cdots$ such that any two trajectories will meet at all but finitely many of these times. Using the Markov property of the conditioned chain, this allows us to couple trajectories of the conditioned chain started at any two initial states. Thus the tail of the conditioned extended chain is trivial, and the exchangeable $\sigma$-field is generated by $V$. 
$\mathrm{R}$ e $\mathrm{m}$ a $\mathrm{rk}$. We can also give a more elementary, but longer, proof of the previous theorem that avoids considering the extended chain conditioned on $V$. Instead, one can verify that for every $k \geqslant 1$, the conditional expectation $\mathbf{E}(f \mid \mathcal{E})$ of any function $f$ that depends on $S_{0}, \ldots, S_{k}$, given the exchangable $\sigma$-field $\mathcal{E}$, is $\sigma(V)$-measurable.

\section{Concluding remarks}

1. For random walk with mean 0 and bounded jumps in $\mathbf{Z}^{d}$, the argument in Section 2 actually implies that the number of approximate spheres that are visited only once by $\left\{S_{1}, \ldots, S_{n}\right\}$, and never revisited by the RW, grows logarithmically in $n$. Following our work, G. Lawler in a recent preprint («Cut Times for Simple Random Walk» (1995)) has refined the methods in his book, and showed that for simple random walk in $\mathbf{Z}^{3}$, the total number of cutpoints among $\left\{S_{1}, \ldots, S_{n}\right\}$ grows like a power of $n$. Thus the cutpoints described in our proof of Proposition 2.1 are non-typical.

2. Consider a transient Markov chain on a tree (i.e., a countable acyclic graph), where all permitted transitions are to nearest neighbors. James [8] shows that for such chains, the exchangeable $\sigma$-field is always generated by the occupation numbers, although for some of these chains the number of cutpoints is finite almost surely. Thus the existence of infinitely many cutpoints is sufficient, but not necessary, for the description of the exchangeable $\sigma$-field in Proposition 1.1.

3. The following question, due to Kaimanovich and Vershik (see [11], $[10])$, is still open.

Question. Let $\left\{S_{n}\right\}$ be a transient Markov chain on the state space $X$. Let $V_{n}(x)$ and $V(x)$ denote the visit counts and occupation numbers defined in the introduction. When is the tail of the chain $\left\{\left(S_{n}, V_{n-1} \bmod 2\right)\right\}$ generated by the random variables $\{V(x) \bmod 2: x \in X\}$ ?

Kaimanovich [10] gives a positive answer to this question when $\left\{S_{n}\right\}$ is a random walk on $\mathbf{Z}$ with non-zero drift. However, even the case where $\left\{S_{n}\right\}$ is simple random walk on $\mathbf{Z}^{3}$ is unresolved.

Acknowledgement. We are grateful to V. Kaimanovich for helpful discussions and for directing our attention to the exchangeable $\sigma$-field. We also thank P. Diaconis and G. Lawler for the references to [4] and [14], respectively.

\section{REFERENCES}

1. Aldous D., Pitman J. On the zero-one law for exchangeable events. - Ann. Probab., 1979 , v. 7, p. $704-723$.

2. Blackwell D., Freedman D. The tail $\sigma$-field of a Markov chain and a theorem of Orey. - Ann. Math. Statist., 1964, v. 35, p. 1291-1295.

3. Burdzy C. Labyrinth dimension of Brownian trace. - Preprint, 1994. 
4. Diaconis P., Freedman D. De Finetti's theorem for Markov chains. - Ann. Probab., 1980 , v. 8, p. $115-130$.

5. Gromov M. Groups of polynomial growth and expanding maps. - Publ. Math. IHES, 1981 , v. 53 , p. $53-78$.

6. Hebisch W., Saloff-Coste L. Gaussian estimates for Markov chains and random walks on groups. - Ann. Probab., 1993, v. 21, p. 673-709.

7. Hewitt E., Savage L. J. Symmetric measures on Cartesian products. - Trans. Amer. Math. Soc., 1955, v. 80, p. 470-501.

8. James $N$. Exchangeable events without cutpoints. - Preprint, 1995.

9. Кайманович $B$. $A$. Полное описание хвостовой $\sigma$-алгебры случайных блужданий и близкие вопросы. - Теория вероятн. и ее примен., 1985, т. 30, в. 1, с. 189-190.

10. Kaimanovich V.A. Poisson boundaries of random walks on discrete solvable groups. - In: Probability Measures on Groups./ Ed. by H. Heyer. New York: Plenum Press, 1991, p. 205-238.

11. Kaimanovich V.A., Vershik A. M. Random walks on discrete groups: Boundary and entropy. - Ann. Probab., 1983, v. 11, p. 457-490.

12. Kochen S., Stone C. A note on the Borel-Cantelli lemma. - Illinois J. Math., 1964, v. 8, p. 248-251.

13. Lawler G. Intersections of Random Walks. Boston: Birkhäuser, 1991.

14. Lawler G., Polaski $T$. Harnack inequalities and difference estimates for random walks with infinite range. - J. Theor. Probab., 1993, v. 6, p. 781-802.

15. Ney $P$., Spitzer $F$. The Martin boundary for random walk. - Trans. Amer. Math. Soc., 1966, v. 121, p. 116-132.

16. Palacios José-Luis. The exchangeable sigma-field of Markov chains. - Z. Wahrscheinlichkeitstheor. verw. Geb., 1985, B. 69, S. 177-186.

17. Spitzer $F$. Principles of Random Walk. New York: Springer-Verlag, 1976.

18. Varopoulos $N$. Th. Théorie du potentiel sur des groupes et des variétés. - C. R. Acad. Sci. Paris. Ser. I, 1986, v. 302, p. 203-205.

19. Varopoulos $N$. Th. Convolution powers on locally compact groups. - Bull. Sci. Math. (2) 1987 , v. 111 , p. $333-342$. 